\title{
Finitary Functors: From Set to Preord and Poset
}

\author{
Adriana Balan ${ }^{1, \star}$ and Alexander Kurz ${ }^{2}$ \\ 1 University Politehnica of Bucharest, Romania \\ 2 University of Leicester, UK
}

\begin{abstract}
We investigate how finitary functors on Set can be extended or lifted to finitary functors on Preord and Poset and discuss applications to coalgebra.
\end{abstract}

Keywords: extension, lifting, relator, simulation, (final) coalgebra, exact square, embedding.

\section{Introduction}

Endofunctors $T:$ Set $\rightarrow$ Set play a crucial role in the theory of coalgebras and the rich body of results on them [4 has been exploited over the years to prove results about the category Coalg $(T)$, and about logics for $T$-coalgebras, uniformly in the functor $T$.

Not as dominant as Set-functors, functors on preorders and on posets have made their appearance, for example, if one is interested in simulation rather than only bisimulation 8 812 . Moreover, we think of the categories Preord and Poset as the natural link between universal coalgebra 14] and domain theory [1, as domains are special posets.

A general plan of work would be the comprehensive study of Preord- and Poset-functors and their relationship to Set-functors and to coalgebras. In this paper, we restrict ourselves to the modest approach of transforming Set-functors into Preord and Poset-functors and study how some properties important from the coalgebraic point of view are transfered. Two notions arise here: extension and lifting of a Set-functor $T$, where extension means a functor which coincides with $T$ on discrete set and lifting means that underlying sets are kept but some order is added.

For extensions, the final coalgebra is discrete, but nevertheless the associated notion of (bi)simulation on posets can be interesting. For liftings, the order on the final coalgebra is similarity, an insight going back to [13, Thm 4.1] and [17, Thm 5.9].

We start from the observation that every finitary Set-functor $T$ has a (canonical) presentation as a coequalizer of two polynomial functors. Since sets are discrete preorders, this coequalizer can be computed in preorders (or posets) to yield a functor $\tilde{T}$ : Preord $\rightarrow$ Preord, which simultaneously lifts and extends $T$. As shown in [16] this leads to interesting examples: if $T$ is the finite powerset

^ Supported by the CNCSIS project PD-56 no. 19/03.08.10.

A. Corradini, B. Klin, and C. Cîrstea (Eds.): CALCO 2011, LNCS 6859, pp. 85-99, 2011.

(C) Springer-Verlag Berlin Heidelberg 2011 
functor, then $\tilde{T}$ on Poset yields the finite(ly generated) convex powerset functor. On the other hand, the final $\tilde{T}$-coalgebra is always discrete and, therefore, does not capture a notion of simulation.

So we study quotients of polynomial functors, but now with ordered coefficients and show that this is equivalent to the notion of an order $\bar{T}:$ Set $\rightarrow$ Preord on a functor $T$ from 8 . The latter investigate conditions under which $\bar{T}$ can be lifted to a functor $\hat{T}$ : Preord $\rightarrow$ Preord. On the other hand, again interpreting the coequalizer of polynomial functors in Preord, we obtain another lifting $\check{T}:$ Preord $\rightarrow$ Preord, which always exists. We show $\hat{T}=\check{T}$ under the conditions which ensure the existence of $\hat{T}$. The table below summarizes the notation for the various extensions and liftings met in the paper.

\begin{tabular}{|l|}
\hline$T:$ Set $\rightarrow$ Set \\
\hline $\bar{T}:$ Set $\rightarrow$ Preord (Def. 9) \\
\hline$\tilde{T}:$ Preord $\rightarrow$ Preord Extension (3.2) \\
\hline$\hat{T}, \check{T}:$ Preord $\rightarrow$ Preord Liftings (Def.-Prop. [16, resp. Def. 24) \\
\hline
\end{tabular}

The last section of the paper focuses on Poset-functors obtained from the previous constructions by taking quotients, with similar results obtained.

Finally, further topics pursued in the paper are the preservation of exact squares (the ordered analogue of weak pullbacks) and of embeddings. The latter is motivated by the result on the expressiveness of modal logic over posets 9, while the former comes from the fact that it replaces preservation of weak pullbacks as a condition for the existence of the relation lifting on preorders or posets [5].

\section{Preliminaries}

We denote by Preord and Poset the categories of preordered sets and of posets, respectively, and monotone maps. We write $D \dashv U$ : Preord $\rightarrow$ Set for the adjunction between the discrete and the forgetful functor. As $U$ has also a right adjoint (which endows a set with the indiscrete preorder), it preserves all limits and colimits. In particular, coequalizers in Preord are computed as in Set, namely for any pair of monotone maps

$$
(X, \leq) \Longrightarrow(Y, \leq) \stackrel{\pi}{\longrightarrow} Z,
$$

their Set-coequalizer $Z$, with the smallest preorder such that $\pi$ is monotone, becomes the coequalizer in Preord. We denote by $Q \dashv J$ : Poset $\rightarrow$ Preord the adjunction between the quotient functor (sending every preordered set to the quotient poset obtained by identifying all $x, y$ with $x \leq y$ and $y \leq x$ ) and the inclusion functor. For later use, recall that coequalizers in Poset are computed in two steps: first, take the coequalizer in Preord, then quotient it to obtain a poset. 
An embedding in Preord or Poset is an injective, monotone and order reflecting map. In both categories, the embeddings are precisely the strong monomorphisms. An exact square [7] in Preord or in Poset is a diagram

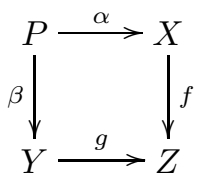

with $f \alpha \leq g \beta$, such that

$$
\forall x \in X, y \in Y . f(x) \leq g(y) \Rightarrow \exists p \in P . x \leq \alpha(p) \wedge \beta(p) \leq y .
$$

If $P$ is $\{(x, y) \in X \times Y \mid f(x) \leq g(y)\}$ with the product order and $\alpha$ and $\beta$ are the usual projections, then (2.2) is obviously satisfied. (2.1) is then called a comma square.

The terminology is borrowed from [7], where exact squares where introduced in the framework of 2-categories. See also [11, where equalizers were similarly replaced by subequalizers. In Set, an exact square is precisely a weak pullback. In [12, a commutative square having the property (2.2) is called a preorder quasi-pullback.

Let $T$ be a Set-functor. It is well-known that $T$ is finitary (commutes with $\omega$-filtered colimits) if and only if it admits a coend representation

$$
T X \cong \int^{n<\omega} \operatorname{Set}(\underline{n}, X) \bullet T \underline{n},
$$

that is, $T X$ has a presentation given by the coequalizer

$$
\coprod_{m, n<\omega} \operatorname{Set}(\underline{m}, \underline{n}) \times T \underline{m} \times X^{n} \underset{\lambda_{X}}{\stackrel{\rho_{X}}{\rightleftarrows}} \coprod_{n<\omega} T \underline{n} \times X^{n} \stackrel{\pi_{X}}{\longrightarrow} T X,
$$

where $\underline{n}$ refers to $\{0,1, \ldots, n-1\}$, and the pair $\left(\lambda_{X}, \rho_{X}\right)$ is given by $\lambda_{X}(f, \sigma, x)=$ $(T f(\sigma), x)$ and $\rho_{X}(f, \sigma, x)=(\sigma, x \circ f)$, for $f: \underline{m} \rightarrow \underline{n}, x: \underline{n} \rightarrow X$ and $\sigma \in T \underline{m} \underline{1}$ Also, $\pi_{X}(\sigma, x)=T x(\sigma)$. Intuitively, $T \underline{n}$ can be seen as the set of operations of arity $n$ applied to the variables in $X$. In the sequel, we shall omit the subscript $X$ when referring to the maps $\lambda_{X}, \rho_{X}$ and $\pi_{X}$ if the context is clear.

\section{From Set to Preord}

Section 3.1 considers the notions of extensions and liftings. Section 3.2 introduces the preordification $\tilde{T}$ of a Set-functor $T$ based on the presentation of $T$. Section 3.3 shows that putting an order on the coefficients of the presentation

${ }^{1}$ We shall identify functions $x: \underline{n} \rightarrow X$ with tuples $x=\left(x_{0}, \ldots, x_{n-1}\right)$, where $x_{i}=$ $x(i), \forall i \in\{0, \ldots, n-1\}$. 
agrees with the notion of an order $\bar{T}$ on a Set-functor $T$ from 8 . Section 3.4 recalls how 8 uses relation lifting to extend $\bar{T}$ to an endofunctor $\hat{T}$ on Preord and shows that if $T$ preserves weak pullbacks then $\tilde{T}$ preserves exact squares and, therefore, embeddings. Section 3.5 emphasizes that the order on the final $\hat{T}$-coalgebra coincides with the similarity given by relation lifting. Whereas [8] use relation lifting to extend $\bar{T}$ to a functor $\hat{T}$ on preorder, we can now also use the presentation to extend $\bar{T}$ to a functor $\check{T}$ on preorders. Section 3.6 shows that $\check{T}=\hat{T}$ under the conditions given in [8] for the existence of $\hat{T}$.

\subsection{Extension and Lifting}

Definition 1. Let $T$ be a Set-functor. An extension of $T$ to Preord is a locally monotone functor $\Gamma:$ Preord $\rightarrow$ Preord such that $\Gamma D=D T$. A lifting of $T$ to Preord is a locally monotone functor $\Gamma:$ Preord $\rightarrow$ Preord such that $U \Gamma=T U$.

In the following, if $T$ is finitary, we also require $\Gamma$ (extension or lifting) to be so 2 Extensions and liftings of a Set-functor to Poset are defined similarly.

It follows that both a lifting and an extension will satisfy the relation $T=$ $U \Gamma D$. Intuitively, an extension will coincide with $T$ on discrete sets, while a lifting means that we put a preorder (respectively a partial order) on $T X$. Also, there is an immediate test to decide whether a (finitary) locally monotone Preord (or Poset)-functor $\Gamma$ is a lifting or an extension of a Set-functor: namely, compute $T=U \Gamma D$ and check if $\Gamma D=D T$ or $U \Gamma=T U$.

Remark 2. An extension is not necessarily unique. Let $\Gamma$ be the functor sending a preordered set (a poset) to the (discrete) set of its connected components. Then $U \Gamma D=I d^{3}$. But also the identity on Preord (respectively on Poset) is an extension of $I d$, showing that a Set-functor can have different extensions.

The local monotonicity requirement is natural, as the categories Preord and Poset are enriched over themselves (in the sense that the hom-sets are ordered) and enriched functors coincide with locally monotone ones. In all constructions that we shall perform, the local monotonicity of the lifted/extended functor will come for free.

Note that although Set is (discretely) enriched over Preord (and over Poset), the adjunction $D \dashv U$ is not, since $U$ is not locally monotone. In particular, $\Gamma=D T U$ will not in general be an extension/lifting and we shall replace it by (3.2) instead.

\footnotetext{
${ }^{2}$ An extension $\Gamma$ of a finitary functor $T$ need not be finitary: consider the finitary functor $T X=\{l: \mathbb{N} \rightarrow X \mid l(n)=l(n+1)$ for all but a finite number of $n\}$. It admits the Preord-extension $\Gamma\left(X, \leq_{X}\right)=\left\{l:\left(\mathbb{N}, \leq_{\mathbb{N}}\right) \rightarrow\left(X, \leq_{X}\right) \mid l(n) \leq\right.$ $l(n+1)$ for all but a finite number of $n\}$. But this $\Gamma$ is not finitary: take the family of finite sets $(\underline{n}, \leq)$ ordered as usual, with inclusion maps, whose colimit is $(\mathbb{N}, \leq \mathbb{N})$. Then one can check that $\operatorname{colim} \Gamma(\underline{n}, \leq) \nRightarrow \Gamma(\operatorname{colim}(\underline{n}, \leq))$. We would like to thank the anonymous referee for pointing us this example. We didn't succeed in constructing a similar example for liftings.

${ }^{3}$ Here $I d$ stands for the identity functor.
} 
If $T$ is a (finitary) Set-functor and $\Gamma$ is an extension of $T$, then $T$-coalgebras and $\Gamma$-coalgebras are related by an adjunction $\tilde{C} \dashv \tilde{D}: \operatorname{Coalg}(T) \rightarrow \operatorname{Coalg}(\Gamma)$ which can be easily derived from the adjunction $C \dashv D$ : Set $\rightarrow$ Preord between the connected components functor and the discrete functor, using that $T=C \Gamma D$ if $\Gamma$ is an extension of $T 4$ Consequently, $\tilde{D}$ will preserve limits, in particular, the final coalgebra (if it exists).

Proposition 3. For any (finitary) extension $\Gamma$ of a finitary functor $T$, the final $\Gamma$-coalgebra does exist and is discrete.

The situation slightly changes when we consider a lifting instead of an extension. In this case there is an obvious forgetful functor $\tilde{U}: \operatorname{Coalg}(\Gamma) \rightarrow \operatorname{Coalg}(T)$, which has a left adjoint still denoted $\tilde{D}: \operatorname{Coalg}(T) \rightarrow \operatorname{Coalg}(\Gamma)$ (this is not hard to check). Thus $\tilde{U}$ preserves limits; in particular the underlying set of the final $\Gamma$-coalgebra (if it exists) will be the final $T$-coalgebra.

Proposition 4. For any (finitary) lifting $\Gamma$ of a finitary functor $T$, the final $\Gamma$-coalgebra exists [10] and is built on the same set as the final T-coalgebra.

\subsection{First Construction: Order on the Variables}

Going back to (2.3), and following [16], we are now interested in this coequalizer if we replace the set $X$ by a preorder $(X, \leq)$. The other sets involved in (2.3) remain discretely ordered, except for $X^{n}$, which carries the product order from $(X, \leq)$. Then $\lambda, \rho$ are monotone. The coequalizer in Preord of this monotone pair of maps $(\lambda, \rho)$ has the same underlying set $T X$, but now with a preorder $\unlhd$ :

$$
\coprod_{m, n<\omega} \operatorname{Set}(\underline{m}, \underline{n}) \times T \underline{m} \times\left(X^{n}, \leq\right) \stackrel{\rho}{\underset{\lambda}{\longrightarrow}} \coprod_{n<\omega} T \underline{n} \times\left(X^{n}, \leq\right) \stackrel{\pi}{\longrightarrow}(T X, \unlhd) .
$$

If $f:(X, \leq) \rightarrow(Y, \leq)$ is a monotone map, it follows that $T f:(T X, \unlhd) \rightarrow$ $(T Y, \unlhd)$ is monotone. Thus we obtain a functor which is also locally monotone

$$
\tilde{T}: \text { Preord } \rightarrow \text { Preord, } \tilde{T}(X, \leq)=(T X, \unlhd) .
$$

It simultaneously defines an extension and a lifting of $T$. In fact, it is an enriched coend $\tilde{T}(X, \leq) \cong \int^{n<\omega}[D \underline{n},(X, \leq)] \bullet D T \underline{n}$, where $[D \underline{n},(X, \leq)]$ refers to the preordered set (internal hom) of all monotone maps from $D \underline{n}$ to $X$.

A functor may have different presentations, 5 but we have

Proposition 5. $\tilde{T}$ is independent of the chosen presentation of $T$.

Example 6. 1. Let $T=\mathcal{P}_{f}$, the finite powerset functor. For $(X, \leq)$ a preordered set, the above construction leads to the Egli-Milner preorder on $\mathcal{P}_{f} X: u \unlhd v$ for $u, v \subseteq X$ finite iff $\forall a \in u \exists b \in v . a \leq b$ and $\forall b \in v \exists a \in u$. $a \leq b$.

${ }^{4}$ We leave the details to the reader. Notice there is a similar adjunction for Posetextensions.

${ }^{5}$ For example, the finite powerset functor $\mathcal{P}_{f}$ can be presented as in (2.3), but also as a quotient of the list-functor $\coprod_{n<\omega} X^{n}$. 
2. Take $T X=1+X$ the lift functor. For $(X, \leq)$ a preordered set, the corresponding order $\unlhd$ on $1+X$ will be the coproduct order.

3. For the list functor $T X=X^{*}$, a preorder on lists is obtained as follows:

$$
\left[x_{0} \ldots x_{n-1}\right] \unlhd\left[y_{0} \ldots y_{m-1}\right] \Leftrightarrow m=n \wedge x_{i} \leq y_{i}, \forall i<n
$$

In Sect. 3.4 we will see another description of $\unlhd$ based on relation liftings.

\subsection{Second Construction: Order on the Operations}

We now equip a functor $T$ with an order on the coefficients of its presentation.

Definition 7. Let $T$ be a finitary Set-functor. We say that $T$ has a presentation with a preorder, if for each finite arity $n$, there is a preorder $\leq$ on $T \underline{n}$ such that $T f:(T \underline{m}, \leq) \rightarrow(T \underline{n}, \leq)$ is monotone for all $f: \underline{m} \rightarrow \underline{n}$.

There are many functors who carry a natural order, as eg the powerset functor (with the inclusion order), or the lift functor $T X=\{\perp\}+X$, with the flat order $\perp \leq x, \forall x \in X$ (see Example 11). The latter is a special case of the following:

Example 8. Let $T$ be a (finitary) Set-functor, but not the constant functor mapping everything to the empty set. Then $T 1 \neq \emptyset$. Specify a preorder on $T 1$. This will induce a preorder on $T \underline{n}$ for all $n<\omega$ via the image of the map $\underline{n} \rightarrow 1$ through $T$, namely the preorder on $T \underline{n}$ is the inverse image of the order on $T 1$. Then we obtain an order on $T$.

Definition 9. Let $T$ be a finitary functor with preorder $\leq$. Consider on $T X$ the preorder $\sqsubseteq$ obtained from the coequalizer

$$
\coprod_{m, n<\omega} \operatorname{Set}(\underline{m}, \underline{n}) \times(T \underline{m}, \leq) \times X^{n} \stackrel{\rho}{\underset{\lambda}{\longrightarrow}} \coprod_{n<\omega}(T \underline{n}, \leq) \times X^{n} \stackrel{\pi}{\longrightarrow}(T X, \sqsubseteq) .
$$

This defines a functor $\bar{T}:$ Set $\rightarrow$ Preord, $\bar{T} X=(T X, \sqsubseteq)$.

Notice that $\rho$ is always monotone, while $\lambda$ is so by Def. 7]. Therefore it makes sense to compute the above coequalizer in Preord. The functor $\bar{T}$ is finitary and satisfies $U \bar{T}=T$. In [8], such a functor is called an order on $T$. We keep the same terminology. This means that on each $T X$ there is a preorder $\sqsubseteq$, and these preorders must be preserved by renaming: for each map $f: X \rightarrow Y$, its image $T f:(T X, \sqsubseteq) \rightarrow(T Y, \sqsubseteq)$ is monotone. Choosing $\underline{n}$ for $X$, we find

Proposition 10. $(T \underline{n}, \sqsubseteq)=(T \underline{n}, \leq)$.

Example 11. 1. Take all T $\underline{n}$ to be discretely ordered. Then $T$ automatically satisfies Def. 7. The preorder obtained on $T X$ will be the discrete one, as any coequalizer of discrete preordered sets is again discrete ( $D$ preserves all colimits being left adjoint, in particular coequalizers). 
2. Take all $T \underline{n}$ to be indiscretely ordered. If $T$ is finitary then all $(T X, \sqsubseteq)=$ $T X \times T X$ are again indiscrete. Indeed, take any $u, v \in T X$. As $T$ is finitary, we can find a finite set $\underline{n}$ and an injection $x: \underline{n} \rightarrow X$ such that $u, v$ lie in the image of the map $T \bar{x}: T \underline{n} \rightarrow T X$. So $u=T x(\sigma)$ and $v=T x(\tau)$ with $\sigma, \tau \in T \underline{n}$. As $T x$ is monotone and $\sigma, \tau$ are comparable, it follows that $u \sqsubseteq v$.

3. Let $T=\mathcal{P}_{f}$ be the finite powerset functor, with the inclusion order on $\mathcal{P}_{f}(\underline{n})$. Then the resulting order on any $\mathcal{P}_{f} X$ is again the inclusion: take any finite subsets $u, v \subseteq X$. Then $u \sqsubseteq v$ if we can find $\sigma \subseteq \underline{n}, x: \underline{n} \rightarrow X, \tau \subseteq \underline{m}$, $y: \underline{m} \rightarrow X$ such that $\pi(\sigma, x)=u, \pi(\tau, y)=v$, and $(\sigma, x)$ and $(\tau, y)$ are comparable in $\coprod_{n<\omega} \mathcal{P}_{f}(\underline{n}) \times X^{n}$. But this can be possible only if both lie in the same component, so $m=n$, and share same variables, $x=y$. It follows $\sigma \subseteq \tau$, hence $u=\mathcal{P}_{f}(x)(\sigma) \subseteq \mathcal{P}_{f}(x)(\tau)=v$. Similarly, if on $\mathcal{P}_{f}(\underline{n})$ we consider the converse inclusion, the resulting preorder $\sqsubseteq$ is $\subseteq$.

4. Take now $T X=\{\perp\}+X$. On the associated signature $\bar{T} \underline{n}=\{\perp\}+\underline{n}$ consider the flat order $\perp<i, \forall i<n$.

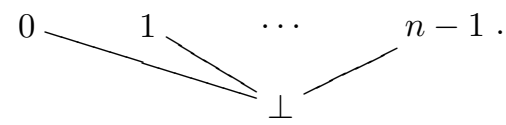

The quotient function $\pi: \coprod_{n<\omega} T \underline{n} \times X^{n} \rightarrow T X$ maps $(\perp, x: n \rightarrow X)$ to $\perp$ and $(i, x: n \rightarrow X)$ to $x_{i}$. It follows now easily that $\perp$ will be the least element in $(\{\perp\}+X, \sqsubseteq)$ and that different elements of $X$ are not comparable, hence on $T X$ we get the same flat order.

5. Consider the polynomial functor $T X=\mathbb{N} \times X$. On each $\mathbb{N} \times \underline{n}$, take the preorder given by $(N, i) \leq(M, j) \Leftrightarrow N<M$ or $(N=M$ and $i=j)$. This is precisely the lexicographic order with respect to the usual ordering of $\mathbb{N}$, when $\underline{n}$ is considered discrete. Then the induced preorder on the quotient $T X$ is similar: two pairs $(N, x)$ and $(M, y)$ in $\mathbb{N} \times X$ are comparable if either are equal or the first components are comparable. In the future, we shall denote this functor by $T X=\mathbb{N} \ltimes X$ to emphasize the special preorder.

6. Let $T=(-)_{2}^{3}$ be the functor introduced by Aczel and Mendler in [2], given on objects by $X_{2}^{3}=\left\{x=\left(x_{1}, x_{2}, x_{3}\right) \in X^{3}||\left(x_{1}, x_{2}, x_{3}\right) \mid \leq 2\right\}$. There is a natural (pre)ordering $\sqsubseteq$ on $X_{2}^{3}$ as follows: all triples $\left(x_{1}, x_{2}, x_{3}\right) \in X_{2}^{3}$ with equal components are minimal elements, all the others are maximals, and a minimal element is comparable with a maximal one only if they share a common component, as in the picture below:

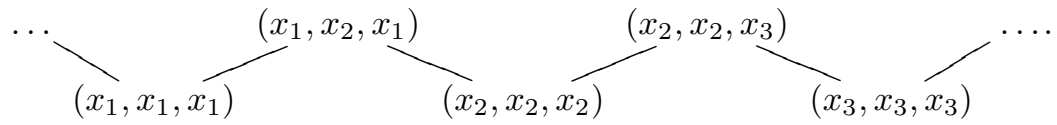

We shall call this the zig-zag preorder. If we restrict it to $T \underline{n}$ and compute $(T X, \sqsubseteq)$ as in (3.3), we obtain again precisely the zig-zag order.

7. Take the list functor $T X=X^{*}$. Put on each $T X$ the following order

$$
\left[x_{0} \ldots x_{n-1}\right] \leq\left[y_{0} \ldots y_{m-1}\right] \Leftrightarrow \exists \varphi: \underline{n} \rightarrow \underline{m} . x_{i}=y_{\varphi(i)}, \forall i<n,
$$


see [8, Example 2.2.(3)], where the function $\varphi$ is required to be strictly monotone. It means that two lists are comparable if one can be obtained from the other by removing some elements. In particular, two lists of same length are comparable only if they are equal. By a similar reasoning as in the previous examples, restricting this preorder to all $T \underline{n}$ and computing $\sqsubseteq$ gives the same order.

The previous examples suggest a correspondence between orders $\bar{T}$ on $T$ and preorders on the associated signature as in Def. [7. This is indeed the case.

Proposition 12. Let $T$ be a finitary Set-functor. Then there is a bijective correspondence between orders on $T$ and presentations with preorders.

\subsection{Lifting $T$ to $\hat{T}$ Using Relators}

Starting from an order $\bar{T}$ on $T$, we will see in this section how a weak pullback preserving functor $T$ lifts to a Preord-endofunctor $\hat{T}$ using relators. We present below a very brief overview on relators, for more details we refer to [15] or [8].

Let $T$ be a Set-functor. For two sets $X, Y$ and a relation $R \subseteq X \times Y$, the $T$-relation lifting of $R$ is

$$
\operatorname{Rel}_{T}(R)=\left\{(u, v) \in T X \times T Y \mid \exists w \in T R . T \pi_{1}(w)=u \wedge T \pi_{2}(w)=v\right\}
$$

where $\pi_{1}, \pi_{2}$ are the projections $X \stackrel{\pi_{1}}{\longleftarrow} R \stackrel{\pi_{2}}{\longrightarrow} Y$. The relation lifting satisfies the following properties $([15])$ :

1. Equality: $=_{T X}=\operatorname{Rel}_{T}\left(=_{X}\right)$.

2. Inclusion: if $R \subseteq S$ then $\operatorname{Rel}_{T}(R) \subseteq \operatorname{Rel}_{T}(S)$.

3. Composition: if $R \subseteq X \times Y$ and $S \subseteq Y \times Z$, then $\operatorname{Rel}_{T}(S \circ R) \subseteq \operatorname{Rel}_{T}(S) \circ$ $\operatorname{Rel}_{T}(R)$, with equality if and only if $T$ preserves weak pullbacks.

4. Inverse images (substitution): given functions $f: X \rightarrow X^{\prime}, g: Y \rightarrow Y^{\prime}$ and relation $R^{\prime} \subseteq X^{\prime} \times Y^{\prime}$, then

$$
\operatorname{Rel}_{T}\left((f \times g)^{-1}\left(R^{\prime}\right)\right) \subseteq(T f \times T g)^{-1}\left(\operatorname{Rel}_{T}\left(R^{\prime}\right)\right)
$$

with equality if $T$ preserves weak pullbacks.

An immediate consequence is the following: if $T$ preserves weak pullbacks and $\leq$ is a preorder on a set $X$, then $\operatorname{Rel}_{T}(\leq)$ is a preorder on $T X$.

Proposition 13. Let $T$ be a finitary Set-functor which preserves weak pullbacks. Then for each preordered set $(X, \leq), \operatorname{Rel}_{T}(\leq)$ coincides with the preorder $\unlhd$ on $T X$ constructed in Sect. 3.2 6

\footnotetext{
${ }^{6}$ Notice that the only thing needed in Prop 13 is that $\operatorname{Rel}_{T}(\leq)$ is a preorder. This is of course implicit when $T$ preserves weak pullbacks, as mentioned earlier. We do not know if there are examples when $\operatorname{Rel}_{T}(\leq)$ is a preorder, for any preordered set $(X, \leq)$, without requesting $T$ to preserve weak pullbacks.
} 
Assume now that there is an order on $T$, given by $\bar{T} X=(T X, \sqsubseteq)$. For any relation $R \subseteq X \times Y$, the associated relation on $T X \times T Y$ given by

$$
\operatorname{Rel}_{T}(R)=\sqsubseteq \circ \operatorname{Rel}_{T}(R) \circ \sqsubseteq
$$

is usually called a $T$-relator, or lax $T$-relation lifting ([15, 8] ). Equivalently,

$$
(u, v) \in \operatorname{Re} \frac{\sqsubseteq}{T}(R) \Longleftrightarrow \exists w \in T(R) . u \sqsubseteq T \pi_{1}(w) \text { and } T \pi_{2}(w) \sqsubseteq v .
$$

The $T$-relator satisfies the following properties (8], Lemma 4.2):

1. $\sqsubseteq_{T X}=\operatorname{Rel} \check{\bar{T}}\left(=_{X}\right)$.

2. If $R \subseteq S$ then $\operatorname{Rel} \stackrel{\subseteq}{T}(R) \subseteq \operatorname{Rel} \frac{\sqsubseteq}{T}(S)$.

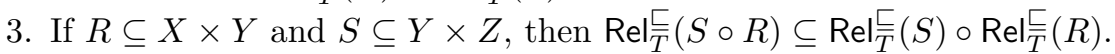

4. For any functions $f: X \rightarrow X^{\prime}, g: Y \rightarrow Y^{\prime}$ and any relation $R^{\prime} \subseteq X^{\prime} \times Y^{\prime}$,

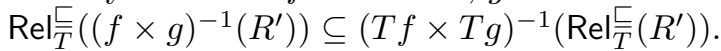

Definition 14. Let $T$ be a Set-endofunctor. We say that an order on $T$, given by $\bar{T}:$ Set $\rightarrow$ Preord, $\bar{T} X=(T X, \sqsubseteq)$,

1. is stable (preserves inverse images) if for any two functions $f: X \rightarrow X^{\prime}$, $g: Y \rightarrow Y^{\prime}$ and any relation $R^{\prime} \subseteq X^{\prime} \times Y^{\prime}$,

$$
\left.\operatorname{Rel}_{\bar{T}}\left((f \times g)^{-1}\left(R^{\prime}\right)\right)=(T f \times T g)^{-1}(\operatorname{Re})_{\bar{T}}^{\sqsubset}\left(R^{\prime}\right)\right) .
$$

2. preserves composition of relations if for any $R \subseteq X \times Y$ and $S \subseteq Y \times Z$,

$$
\operatorname{Rel} \underset{\bar{T}}{\sqsubseteq}(S \circ R)=\operatorname{Rel} \underset{\bar{T}}{\sqsubseteq}(S) \circ \operatorname{Rel}_{\bar{T}}^{\sqsubseteq}(R) .
$$

3. preserves composition of preorders if for any preordered set $(X, \leq)$,

$$
\operatorname{Rel} \underset{\bar{T}}{\sqsubseteq}(\leq) \circ \operatorname{Rel} \stackrel{\sqsubseteq}{\bar{T}}(\leq) \subseteq \operatorname{Rel} \frac{\sqsubseteq}{\bar{T}}(\leq) .
$$

Proposition 15. For any order on $T$, we have (3.5) $\Rightarrow(3.6) \Rightarrow 3.7$.

Now the purpose of all these preparations is the following

Definition-Proposition 16. Let $T$ be a finitary functor having an order $\bar{T}$ which preserves composition of preorders. Then it lifts to a Preord-endofunctor $\hat{T}\left([8]\right.$, Lemma 5.5), given by $\hat{T}(X, \leq)=\left(T X, \operatorname{Rel}_{T}(\leq)\right)$.

The functor $\hat{T}$ is locally monotone (defining thus a lifting in the sense of Def. 1): assume $f, g:(X, \leq) \rightarrow(Y, \leq)$ are monotone maps such that $f \leq g$ pointwise. Then for any $u \in T X$, we have $u \sqsubseteq u$ and

$$
\sqsubseteq=\operatorname{Rel} \frac{\sqsubseteq}{T}\left(=_{X}\right) \subseteq \operatorname{Rel} \frac{\sqsubseteq}{\bar{T}}\left((f \times g)^{-1}(\leq)\right) \subseteq(T f \times T g)^{-1}\left(\operatorname{Rel} \frac{\sqsubseteq}{\bar{T}}(\leq)\right),
$$

therefore $(T f(u), T g(u)) \in \operatorname{Rel}_{T} \stackrel{\sqsubseteq}{\bar{T}}(\leq)$. 
It follows that any finitary functor having an order which preserves composition of preorders has a lifting 7 to Preord. We also point out that having an order which preserves composition of relations (preorders) is not equivalent nor implied by the preservation of weak pullbacks by the functor $T$ itself, unless discrete preorder is involved (see Ex. 17/1 and also Ex. 17/5 and Ex. 17/6). Any polynomial functor has an order which preserves composition of relations (see 8], Def. 4.4 and the following paragraph there), but this property is not necessarily preserved by their quotients (see below Ex. 17/5).

In all examples below, $T$ is a finitary Set-functor.

Example 1\%. 1. Assume the ordering on the operations of $T$ is discrete. Then $\sqsubseteq$ is equality and $\operatorname{Rel} \underset{T}{\leftrightarrows}(R)=\operatorname{Rel}_{T}(R)$. Therefore the order on $T$ is stable iff $T$ preserves weak pullbacks. In this case, the lifting of $T$ to Preord will be $\hat{T}(X, \leq)=\left(T X, \operatorname{Rel}_{T}(\leq)\right)$. In view of Prop. 13, we obtain that $\hat{T}=\tilde{T}$.

2. Assume that the order on the operations of $T$ is indiscrete. We have seen that $\sqsubseteq=T X \times T X$, hence $\operatorname{Rel} \underset{T}{\sqsubseteq}(R)=T X \times T Y$, for any $R \subseteq X \times Y$, provided $\operatorname{Rel}_{T}(R)$ is not empty. Actually, what we only need is that $T(\leq)$ to be non empty, which happens for all (finitary) functors $T$ except for the constant one mapping everything to the empty set. Then property 4 of $T$-relators holds with equality, hence again we get a lifting by $\hat{T}(X, \leq)=(T X, T X \times T X)$.

3. Let $T$ be now the finite power-set functor $\mathcal{P}_{f}$, with inclusion as (pre)order on each $\mathcal{P}_{f} X$. Then for $R \subseteq X \times Y$, $\operatorname{Rel}_{\mathcal{P}_{f} X}(R)$ can be described as follows (see for example [15], Thm. 2.3.2):

$(u, v) \in \operatorname{Re}_{\mathcal{P}_{f} X}(R) \Leftrightarrow \forall a \in u \exists b \in v .(a, b) \in R \wedge \forall b \in v \exists a \in u .(a, b) \in R$.

An easy computation shows now that the order $X \mapsto\left(\mathcal{P}_{f} X, \subseteq\right)$ preserves composition (is even stable), hence $\mathcal{P}_{f}$ lifts to a functor $\hat{\mathcal{P}}_{f}(X, \leq)$ $=\left(\mathcal{P}_{f} X, \operatorname{Rel} \overline{\mathcal{P}}_{f}{ }_{f}(\leq)\right)$ on Preord, with ordering

$$
(u, v) \in \operatorname{Rel}_{\mathcal{P}_{f}}^{\subseteq}(\leq) \Leftrightarrow \forall a \in u \exists b \in v . a \leq b .
$$

4. For $T X=\{\perp\}+X$, The order from Ex. 114 preserves at least composition of preorders, as the relation extension is $\operatorname{Rel}_{T}(R)=R \cup\{(\perp, \perp)\}$. The resulting functor $\hat{T}$ will then add a bottom element to any preordered set $(X, \leq)$.

5. Take now the finitary functor $T X=X_{2}^{3}$. The relation lifting associated to this functor is

$$
\left(\left(x_{1}, x_{2}, x_{3}\right),\left(y_{1}, y_{2}, y_{3}\right)\right) \in \operatorname{Rel}_{(-)_{2}^{3}}(R) \Leftrightarrow\left(\left(x_{1}, y_{1}\right),\left(x_{2}, y_{2}\right),\left(x_{3}, y_{3}\right)\right) \in R_{2}^{3}
$$

for $R \subseteq X \times Y$. It is well-known that this functor does not preserve weak pullbacks. Recall that we have introduced the zig-zag preorder on $T X$ (Ex. 11). This order is not stable nor preserves composition: for stability, take $X=\{0\}, Y=\{1\}$ and $X^{\prime}=Y^{\prime}=\{0,1\}$, with $f, g$ the inclusion

${ }^{7}$ This is no longer an extension: for discrete sets, $\hat{T}(X,=)=(T X, \sqsubseteq)$ is not necessarily discrete. 
maps, and relation $R^{\prime}={ }_{X^{\prime}}$. Then $(T f \times T g)^{-1}(\operatorname{Rel} \underset{T}{\sqsubseteq}(=))=T X \times T Y$, while $\operatorname{Rel} \stackrel{\sqsubseteq}{T}\left((f \times g)^{-1}(=)\right)=\emptyset$. For preservation of composition, take again $X=\{0,1\}$ and the (preorder) relation $R=\{(0,0),(0,1),(1,1)\}$. Then for example $((0,0,1),(0,1,0)) \in \operatorname{Rel}_{(-)_{2}^{3}}^{\sqsubseteq}(R) \circ \operatorname{Rel}_{(-)_{2}^{3}}^{\sqsubseteq}(R)$, but $((0,0,1),(0,1,0)) \notin$ $\operatorname{Rel} \underset{(-)_{2}^{3}}{\sqsubseteq}(R \circ R)=\operatorname{Rel} \underset{(-)_{2}^{3}}{\sqsubseteq}(R)$.

6. For the polynomial functor $T X=\mathbb{N} \ltimes X$ lexicographically ordered, a similar argument to the one in [8] shows that it is not stable. But it preserves composition with respect to preorders: if $(X, \leq)$ is a preordered set, then $\operatorname{Rel} \frac{\check{T}}{\bar{T}}(\leq)$ is again a preorder, namely the usual lexicographic one on $\mathbb{N} \ltimes X$ :

$$
((n, x),(m, y)) \in \operatorname{Rel}_{\bar{T}}^{\sqsubseteq}(\leq) \Leftrightarrow n<m \text { or }(n=m \text { and } x \leq y)
$$

Proposition 18. Let $T$ be a finitary Set-functor having an order $\bar{T}(X, \leq)=$ $(T X, \sqsubseteq)$. Then the following are equivalent:

1. The order is stable.

2. $\bar{T}$ maps weak pullbacks to exact squares.

3. The lifted functor $\hat{T}$ preserves exact squares.

Corollary 19. Let $T$ be a finitary Set-functor which preserves weak pullbacks. Then the Preord-lifting $\tilde{T}$ from (3.2) preserves exact squares.

Intuitively, we could simply say that if a Set-functor $T$ preserves exact squares, then its lifting $\tilde{T}$ does so.

It is well known that all Set-functors preserve injective maps with non-empty domain. In Preord we are more interested in embeddings, and they are preserved if the functor preserves exact squares, see [7. Hence we have the following.

Proposition 20. If the order on $T$ is stable, then $\hat{T}$ preserves embeddings.

Corollary 21. If T preserves weak pullbacks, then $\tilde{T}$ preserves embeddings.

\subsection{Preorder on the Final Coalgebra}

There are several papers in the literature describing order relations on the final $T$-coalgebra (see for example [3] or 8]); as it is expected, there is a connection with the order on the final coalgebra of the lifted functor, first emphasized in [12. This section intends to present a direct approach of that.

We shall assume that $T$ has a stable order $\bar{T} X=(T X, \sqsubseteq)$ and we shall work with the associated lifting $\hat{T}(X, \leq)=(T X, \operatorname{Re} \stackrel{\complement}{\bar{T}}(\leq))$. Recall from $[8$ that a $T$ simulation with respect to the order $\sqsubseteq$ between two coalgebras $X \stackrel{c}{\rightarrow} T X$ and $Y \stackrel{d}{\rightarrow} T Y$ is a relation $R \subseteq X \times Y$ such that $(x, y) \in R \Rightarrow(c(x), d(y)) \in \operatorname{Rel} \underset{\bar{T}}{\sqsubseteq}(R)$. In particular, for any $\hat{T}$-coalgebra $(X, \leq) \stackrel{c}{\rightarrow}(T X, \operatorname{Rel} \underset{\bar{T}}{\sqsubseteq}(\leq))$, the monotonicity of $c$ implies the preorder $\leq$ is a simulation on $X$. The greatest simulation between two coalgebras is called similarity and denoted by $\lesssim$. It satisfies the following (8], Prop. 5.4): 
1. For any $T$-coalgebra homomorphisms $X \stackrel{f}{\rightarrow} Z, Y \stackrel{g}{\rightarrow} W$ and $x \in X, y \in Y$, we have $x \lesssim y \Leftrightarrow f(x) \lesssim g(y)$.

2. Similarity on a coalgebra $X \stackrel{c}{\rightarrow} T X$ is a preorder.

Let now $Z \stackrel{z}{\rightarrow} T Z$ be the final $T$-coalgebra (which exists by the finitarity assumption on $T$ ). As $\hat{T}$ is also finitary and $\tilde{U}$ preserves limits, the final $\hat{T}$-coalgebra is also $Z$, but now with some preorder $\leq_{Z}$ such that $\left(Z, \leq_{Z}\right) \stackrel{z}{\rightarrow}\left(T Z, \operatorname{Rel} \underset{\bar{T}}{\leftrightarrows}\left(\leq_{Z}\right)\right)$ is an isomorphism in Preord. In particular, $\leq_{Z}$ is a simulation, hence $\leq_{Z} \subseteq \lesssim_{Z}$. Take $(X, \leq) \stackrel{c}{\rightarrow}(T X, \operatorname{Rel} \stackrel{\complement}{\bar{T}}(\leq))$ a $\hat{T}$-coalgebra, with (monotone) anamorphism $(X, \leq) \stackrel{!}{\rightarrow}\left(Z, \leq_{Z}\right)$. By property (11) above, $(X, \leq) \stackrel{!}{\rightarrow}\left(Z, \lesssim_{Z}\right)$ is monotone and a $\hat{T}$ - coalgebra map, hence in the following diagram the identity on $Z$ is monotone 8 , implying $\lesssim_{Z} \subseteq \leq_{Z}$.

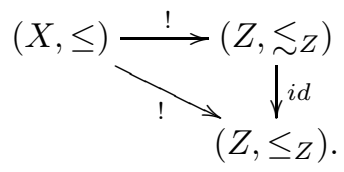

We have thus the following:

Proposition 22. The preorder on the final $\hat{T}$-coalgebra is the similarity 9

Remark 23. By [8], Thm. 6.2, if the order satisfies the condition

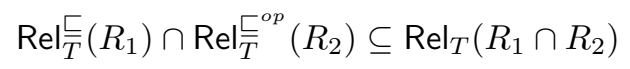

for any two relations $R_{1}, R_{2} \subseteq X \times Y$, then the two-way similarity $\lesssim \cap \lesssim o p$ is the same as bisimilarity. This holds for all coalgebras, in particular for the final coalgebra. But bisimilarity on final coalgebra is equality, hence the above condition implies that similarity on the final coalgebra $Z$ is a partial order.

\subsection{Third Construction: Order the Variables and Operations}

Here we lift an order $\bar{T}$ on $T$ to a Preord-endofunctor $\check{T}$ even in the case that $T$ does not preserve weak pullbacks. The idea is to subsume the constructions in Sect 3.2 and 3.3 in a single one: putting order on the signature (as in Def. (7) and building the coequalizer of (2.3) in Preord.

Definition 24. Let $T$ be a functor with preorder $\sqsubseteq$. Denote by $\check{T}$ the functor given by the coequalizer $(T X, \preceq)$ below

$\coprod_{m, n<\omega} \operatorname{Set}(\underline{m}, \underline{n}) \times(T \underline{m}, \sqsubseteq) \times\left(X^{n}, \leq\right) \stackrel{\rho}{\lambda} \underset{n<\omega}{\Longrightarrow}(T \underline{n}, \sqsubseteq) \times\left(X^{n}, \leq\right) \underset{\pi}{\longrightarrow}(T X, \preceq)$

${ }^{8} \mathrm{As}\left(Z, \lesssim_{Z}\right)$ is a $\hat{T}$-coalgebra, there is unique monotone map from it to the final $\hat{T}$ coalgebra. Via the forgetful functor, this is mapped to the unique $T$-coalgebra map $Z \rightarrow Z$ which is obviously the identity.

${ }^{9}$ See also 13, Thm. 4.1 and 17, Thm. 5.6. 
where the domain and the codomain of the coequalizer pair carry the coproduct preorder and each component of the coproduct has the product preorder $(\operatorname{Set}(\underline{m}, \underline{n})$ is discrete, while $X^{n}$ has the product preorder obtained from the one on $X$ ).

Observe that $\check{T}$ is locally monotone and that $\sqsubseteq \subseteq \preceq$. Moreover, we have

Theorem 25. Let $T$ be a finitary Set-endofunctor which preserves weak pullbacks and has an order which preserves composition of preorders. Then the liftings $\check{T}$ and $\hat{T}$ coincide.

Example 26. The construction presented at the beginning of this section says that we can still get a lifting, independently of $\mathrm{T}$ preserving weak pullbacks or the order preserving composition of relations; for example, consider the functor $T X=X_{2}^{3}$ with the zigzag order. The corresponding preorder $\preceq$ can then be described as follows: for $\left(x_{1}, x_{2}, x_{3}\right),\left(y_{1}, y_{2}, y_{3}\right) \in X_{2}^{3},\left(x_{1}, x_{2}, x_{3}\right) \preceq\left(y_{1}, y_{2}, y_{3}\right)$ if $x_{i} \leq y_{i}, \forall 1 \leq i \leq 3$, or $x_{1}=x_{2}=x_{3}$ and there is some $1 \leq i \leq 3$ with $x_{i} \leq y_{i}$.

\section{From Preord to Poset}

Given a finitary Set-functor $T$, assume that we have an extension (or a lifting) $\Gamma$ to Preord. We can move further to Poset by taking the locally monotone functor $T^{\prime}=Q \Gamma J:$ Poset $\rightarrow$ Preord, where $Q \dashv J$ is the (monadic) adjunction between the quotient and the inclusion functor mentioned in the preliminaries. If $\Gamma$ is finitary, then $T^{\prime}$ is also, since both $J$ and $Q$ preserve filtered colimits 10 Regarding coalgebras, notice that each $\Gamma$-coalgebra can be quotiented to a $T^{\prime}$-coalgebra, thus there is a functor $Q^{\prime}: \operatorname{Coalg}(\Gamma) \rightarrow \operatorname{Coalg}\left(T^{\prime}\right)$, which sends a $\Gamma$-coalgebra $X \stackrel{c}{\rightarrow} \Gamma X$ to $Q X \stackrel{Q c}{\longrightarrow} Q \Gamma X \rightarrow T^{\prime} Q X$.

Now the discussion bifurcates according to $\Gamma$ being an extension or a lifting.

If $\Gamma$ is an extension, a simple computation shows that $T^{\prime}$ maps discrete sets to discrete sets, thus it is a Poset-extension of $T$. Moreover, a similar discussion to the one in Section 3.1 shows that the final $T^{\prime}$-coalgebra exists and is discrete, with same carrier as the final $T$-coalgebra, once we assume $T$ (and $\Gamma$ ) finitary.

For the particular extension $\tilde{T}(X, \leq)=(T X, \unlhd)$ of a finitary functor $T$ from Section 3.2. relations (3.1) and (3.2), let $T^{\prime}(X, \leq)$ be the quotient Poset-functor, whose ordering will be denoted for convenience with same symbol $\unlhd$. In case $T$ preserves weak pullbacks, $\unlhd$ on $T X$ has been expressed in terms of the relation lifting (Prop.13). An immediate result is

Proposition 27. Let $T$ be a finitary weak pullbacks preserving Set-functor and $T^{\prime}=Q \tilde{T} J$ its Poset-extension, with $\tilde{T}$ as in (3.1). Then $T^{\prime}$ preserves exact squares and embeddings.

Remark 28. If $T$ does not preserve weak pullbacks, then $T^{\prime}$ may fail to preserve embeddings, as we can see from the following example: take $T$ to be the functor part of the Boolean algebra monad. On finite sets, we can identify $T$ with the

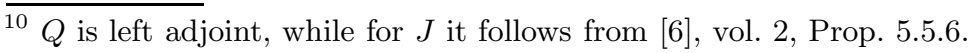


composition of the contravariant power-set functor with itself. Then $T$ does not preserve weak pullbacks [14]. We shall show that the corresponding extension to Poset does not preserve embeddings. For this, take the embedding of the discrete two-elements poset $\{a, b\}$ into the poset $\{a, b, c\}$ ordered by $a<c, b<c$. Then $T^{\prime}(\{a, b\},=)$ is the (discrete) free Boolean algebra on two generators with 16 elements. For the poset (Boolean algebra) $\left(T^{\prime}(\{a, b, c\}, \leq), \unlhd\right)$, notice that monotonicity of operations implies $\perp \unlhd a \wedge \neg a \unlhd a \wedge \neg c \unlhd c \wedge \neg c=\perp$, thus $a \unlhd c$. Similarly, $\perp \unlhd a \wedge \neg a \unlhd c \wedge \neg a \unlhd c \wedge \neg c=\perp$ implies $c \unlhd a$. Thus the images of $a$ and $c$ in $T^{\prime}(\{a, b, c\}, \leq)$ coincide; similarly for the images of $b$ and $c$, which makes us conclude that $T^{\prime}(\{a, b, c\}, \leq)$ has only 4 elements (the free Boolean algebra on only one generator). Hence $T^{\prime}$ cannot preserve the embedding $(\{a, b\},=) \hookrightarrow(\{a, b, c\}, \leq)$.

In case $\Gamma$ is a lifting, there is a preorder on $T X$ for each $(X, \leq)$ and $T^{\prime}(X, \leq)$ is the quotient of $T X$ with respect to the equivalence relation induced by that preorder. The resulting functor $T^{\prime}$ is, in general, no longer a lifting of $T$ to Poset nor an extension.

However, if we consider a particular lifting of $T$, namely $\hat{T}$, with respect to an order $\sqsubseteq$ which is already a partial order, then by restricting to posets we obtain that $\operatorname{Rel} \overline{\bar{T}}(\leq)$ is a partial order on $T X$ (for each poset $(X, \leq)$ ), once we assume that $\sqsubseteq$ preserve compositions of preorders and satisfies (3.8). In this case, $Q \hat{T} J$ can be identified with $\hat{T} J$ and defines a lifting of $T$ to Poset. In the general case, however, the best that we can say is that the analogue of Prop. 10 holds, namely that $T^{\prime}$ will coincide with $T$ on finite sets $\underline{n}$, with partial order $\sqsubseteq$.

Example 29. 1. Let $\mathcal{D}$ be the finite subdistribution functor, $\mathcal{D} X=\{d: X \rightarrow$

$\left.[0,1]\left|\sum_{x \in X} d(x) \leq 1,\right| \operatorname{supp}(d) \mid<\infty\right\}$, with the pointwise order $d \sqsubseteq d^{\prime} \Leftrightarrow$ $d(x) \leq d^{\prime}(x), \forall x \in X$. The corresponding $\hat{\mathcal{D}}$ maps posets to posets (see comments after Def. 11 in [12]).

2. Take now $\mathcal{P}_{f}$, the finite powerset functor, with the inclusion order. Then this time the Poset-functor is indeed a quotient, namely the finite convex power set.

Although $T^{\prime}$ is not a lifting nor an extension, it still behaves well with respect to exact squares (and embeddings):

Proposition 30. Let $T$ be a finitary Set-functor having an order $\bar{T}(X, \leq)=$ $(T X, \sqsubseteq)$ which is stable and $T^{\prime}=Q \hat{T} J$ as above. Then $T^{\prime}$ preserves exact squares, thus also embeddings.

\section{Conclusion}

Considering the rich body of results on Set-functors, see eg [4, our investigations suggest that analogous results for functors on preorders or on posets would be of interest to coalgebra. For example, characterizations of functors that preserve exact squares or embeddings would be of interest. This also links the current paper with investigations on coalgebraic logic over preorders or posets, where 
first steps have been taken on logics given by predicate liftings in 9 and on Moss's $\nabla$ in [5]. Another direction is to follow the connection with coalgebraic (bi)simulations like in 812.

Acknowledgements. We would like to thank J. Velebil for pointing out the importance of exact squares and the reference [7], and the referees for their valuable suggestions.

\section{References}

1. Abramsky, S., Jung, A.: Domain Theory. In: Abramsky, S., Gabbay, D.M., Maibaum, T.S.E. (eds.) Handbook of Logic in Computer Science, vol. 3, pp. 1168. Oxford Univ. Press, New York (1994)

2. Aczel, P., Mendler, N.: A Final Coalgebra Theorem. In: Dybjer, P., Pitts, A.M., Pitt, D.H., Poigné, A., Rydeheard, D.E. (eds.) Category Theory and Computer Science. LNCS, vol. 389, pp. 357-365. Springer, Heidelberg (1989)

3. Adámek, J.: Final Coalgebras Are Ideal Completions of Initial Algebras. J. Logic Computat. 12(2), 217-242 (2002)

4. Adámek, J., Trnková, V.: Automata and Algebras in Categories. Mathematics and Its Applications: East European Series, vol. 37. Kluwer Academic Publishers, Dordrecht (1990)

5. Bilková, M., Kurz, A., Petrişan, D., Velebil, J.: Relation Liftings on Preorders and Posets. In: Corradini, A., Klin, B., Cârstea, C. (eds.) CALCO 2011. LNCS, vol. 6859, pp. 115-129. Springer, Heidelberg (2011)

6. Borceux, F.: Handbook of Categorical Algebra. In: Encycl. Mathem. Appl., vol. 5052. Cambridge Univ. Press, Cambridge (1994)

7. Guitart, R.: Relations et Carrés Exacts. Ann. Sci. Math. Québec 4(2), 103-125 (1980)

8. Hughes, J., Jacobs, B.: Simulations in Coalgebra. Theor. Comput. Sci. 327, 71-108 (2004)

9. Kapulkin, K., Kurz, A., Velebil, J.: Expressivity of Coalgebraic Logic over Posets. In: Jacobs, B.P.F., Niqui, M., Rutten, J.J.M.M., Silva, A.M. (eds.) CMCS 2010 Short contributions, CWI Technical report SEN-1004, pp. 16-17 (2010)

10. Karazeris, P., Matzaris, A., Velebil, J.: Final Coalgebra in Accessible Categories, accepted for publication in Math. Struct. Comput. Sci., http://xxx.lanl.gov/abs/0905.4883

11. Lambek, J.: Subequalizers. Canad. Math. Bull. 13, 337-349 (1970)

12. Levy, P.: Similarity Quotients as Final Coalgebras. In: Hofmann, M. (ed.) FOSSACS 2011. LNCS, vol. 6604, pp. 27-41. Springer, Heidelberg (2011)

13. Rutten, J.: Relators and Metric Bisimulations (Extended Abstract). In: Jacobs, B., Moss, L., Reichel, H., Rutten, J. (eds.) First Workshop on Coalgebraic Methods in Computer Science, CMCS 1998. Electr. Notes Theor. Comput. Sci., vol. 11, pp. 252-258 (1998)

14. Rutten, J.: Universal Coalgebra: A Theory of Systems. Theor. Comput. Sci. 249, 3-80 (2000)

15. Thijs, A.: Simulation and Fixed Point Sematics, Ph. D. Thesis, University of Groningen (1996)

16. Velebil, J., Kurz, A.: Equational Presentations of Functors and Monads. Math. Struct. Comput. Sci. 21(2), 363-381 (2011)

17. Worrell, J.: Coinduction for Recursive Data Types: Partial Orders, Metric Spaces and $\Omega$-Categories. In: Reichel, H. (ed.) Coalgebraic Methods in Computer Science, CMCS 2000. Electr. Notes Theor. Comput. Sci., vol. 33, pp. 337-356 (2000) 